\title{
Which wavelength is the best for arterial pulse waveform extraction using laser speckle imaging?
}

\author{
Pedro Vaz ${ }^{\mathrm{a}, \mathrm{b}, *}$, Tânia Pereira ${ }^{\mathrm{a}}$, Edite Figueiras ${ }^{\mathrm{c}}$, Carlos Correia $^{\mathrm{a}}$, Anne Humeau-Heurtier ${ }^{\mathrm{b}}$, \\ João Cardoso ${ }^{a}$ \\ a LIBPhys-UC, Department of Physics, University of Coimbra, R. Larga, 3004-516 Coimbra, Portugal \\ b Université d'Angers, LARIS - Laboratoire Angevin de Recherche en Ingénierie des Systèmes, 62 avenue Notre-Dame du Lac, 49000 Angers, France \\ ${ }^{\mathrm{c}}$ Tampere University of Technology, BioMediTech, FinnMedi 1, Biokatu 6, 4-211 TTY 33520 Tampere, Finland
}

\section{A R T I C L E I N F O}

\section{Article history:}

Received 14 September 2015

Received in revised form

13 November 2015

Accepted 30 November 2015

Available online 22 December 2015

\section{Keywords:}

Laser speckle

Arterial pulse waveform

Multi-spectral

Correlation

Fast Fourier transform

\begin{abstract}
A B S T R A C T
A multi-wavelengths analysis for pulse waveform extraction using laser speckle is conducted. The proposed system consists of three coherent light sources ( $532 \mathrm{~nm}, 635 \mathrm{~nm}, 850 \mathrm{~nm}$ ). A bench-test composed of a moving skin-like phantom (silicone membrane) is used to compare the results obtained from different wavelengths. The system is able to identify a skin-like phantom vibration frequency, within physiological values, with a minimum error of $0.5 \mathrm{mHz}$ for the $635 \mathrm{~nm}$ and $850 \mathrm{~nm}$ wavelengths and a minimum error of $1.3 \mathrm{mHz}$ for the $532 \mathrm{~nm}$ light wavelength using a FFT-based algorithm. The phantom velocity profile is estimated with an error ranging from $27 \%$ to $9 \%$ using a bidimensional correlation coefficient-based algorithm. An in vivo trial is also conducted, using the $532 \mathrm{~nm}$ and $635 \mathrm{~nm}$ laser sources. The $850 \mathrm{~nm}$ light source has not been able to extract the pulse waveform. The heart rate is identified with a minimum error of 0.48 beats per minute for the $532 \mathrm{~nm}$ light source and a minimal error of 1.15 beats per minute for the $635 \mathrm{~nm}$ light source. Our work reveals that a laser speckle-based system with a $532 \mathrm{~nm}$ wavelength is able to give arterial pulse waveform with better results than those given with a $635 \mathrm{~nm}$ laser.
\end{abstract}

(c) 2015 Elsevier Ltd. All rights reserved.

\section{Introduction}

Laser speckle (LS) is an effect that results from an interference phenomenon and can be characterized as a random pattern of light intensities. Speckle patterns are created when coherent light is reflected by a surface with a rough structure, thus producing random phase variations at different surface locations [1]. The interference between different beams produces a granular pattern of intensities [2]. Historically, this has been considered to be a drawback when using coherent light sources because of the limiting effect on the spatial resolution. To overcome this problem many techniques have been developed [3-5].

Beyond the limitations imposed by speckle, many useful applications of this interferometric effect have been proposed. LS-based techniques are successfully used to estimate two-dimensional blood flow [6,7], to investigate skin vibration [8,9], to measure water flow in plants [10], to assess vibrations modes of remote objects [11], to measure large-object deformations [12] and

\footnotetext{
* Corresponding author at: Department of Physics, University of Coimbra, R. Larga, 3004-516 Coimbra, Portugal. Tel.: +351 239410109.

E-mail address: pvaz@lei.fis.uc.pt (P.Vaz).
}

surfaces movement identification [13]. By using LS pattern analysis, it is also possible to extract information about the target roughness and displacement.

In addition to the possibility of characterizing different materials using LS, the variations of speckle patterns over time (time-varying speckle) can be used to estimate the movement of a specific target [14]. The space-time statistical properties of dynamic speckle patterns depend on the velocity of the target. If the target is static, the speckle pattern does not change in consecutive images [15]. However, if the target is moving, the speckle pattern changes over time and the resulting images can grant information on the original kinetics of the reflecting media [16].

One of the major features of this laser vibrometry technique arises from its truly non-contact nature. This is an unquestionable advantage when compared to other motion assessment techniques that require contact, particularly when the targets are sensitive to mass variations or external pressure [2]. Such is the case of biological systems, like arteries or skin, where the vibration profile can be affected by the forces exerted during the measurement procedure [17].

The aim of the present work is to analyze which wavelength would be the most adequate to extract the arterial pulse waveform using the laser speckle effect. For this purpose, the paper presents 


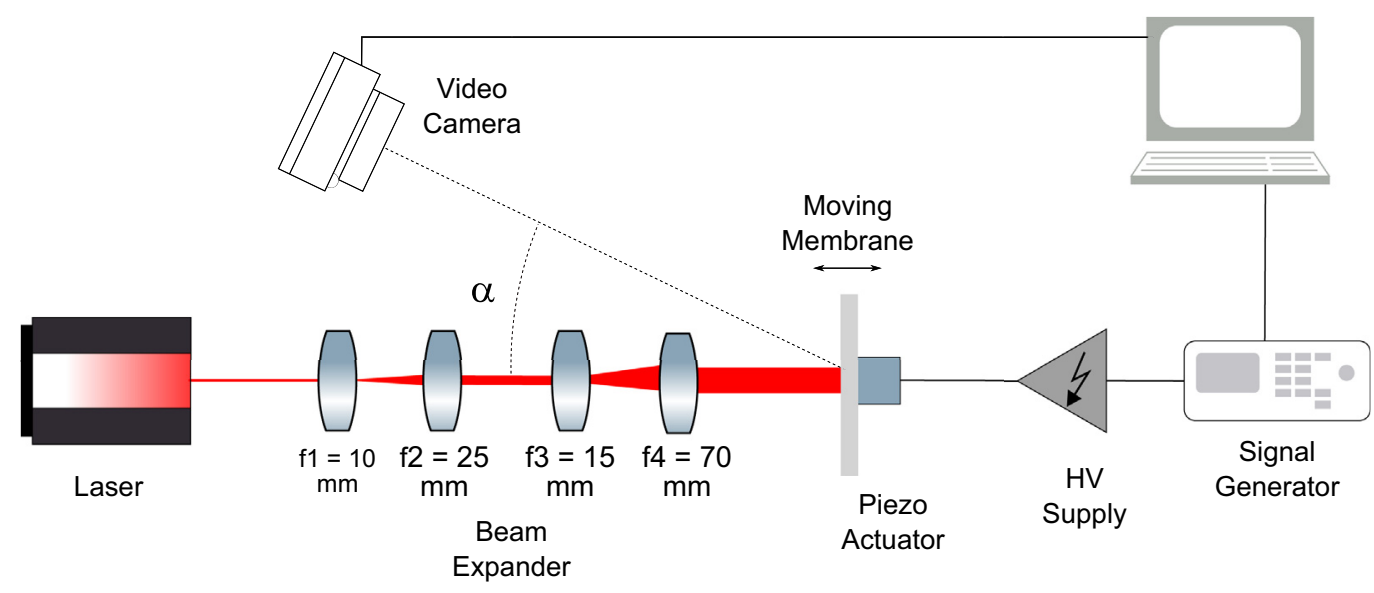

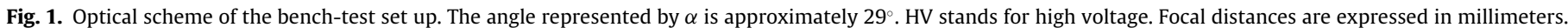
Image not to scale.

an apparatus along with a group of methodologies that can be used to evaluate vibration of skin-like phantoms using 3 different optical wavelengths ( $532 \mathrm{~nm}, 635 \mathrm{~nm}$, and $850 \mathrm{~nm}$ ). LS techniques are now widely used to monitor microvascular blood flow [18]. The detection, with a single system, of information from the macro and the microvascular levels would be of great interest.

The methodology used (correlation coefficient) represents a more efficient computational method, compared to the one used by several authors (cross-correlation) [19-21]. The cross-correlation approach involves, in addition to the shifting of two images along all the possible positions, the identification of the maximum correlation peak. The proposed approach applies a one-dimensional correlation coefficient between two consecutive images. This type of approach was used by Nemati et al. [22,23] but using a different measurement site.

\section{Methods}

\subsection{Experimental set-up}

Speckle patterns were produced by using three distinct lasers: a green laser diode $\left(L_{532}\right)$, CPS532 from Thorlabs, with a wavelength of $532 \mathrm{~nm}$, with an optical power of $4.5 \mathrm{~mW}$, with an output circular beam diameter of $3.5 \mathrm{~mm}$, and with a spectral-width of $0.5 \mathrm{~nm}$; a red laser diode $\left(L_{635}\right)$ (VHK Coherent Inc.) with a wavelength of $635 \mathrm{~nm}$, with an optical power of $4.9 \mathrm{~mW}$, with an output circular beam diameter of $1.1 \mathrm{~mm}$, and a spectral-width of $0.5 \mathrm{~nm}$; and a near infra-red laser diode $\left(L_{850}\right)$, LDL $175 \mathrm{G}$ from Global Lasers, with a wavelength of $850 \mathrm{~nm}$, with an optical power of $3 \mathrm{~mW}$, an output focusable elliptical beam diameter of $4 \times 2 \mathrm{~mm}$, and a spectral-width of $0.5 \mathrm{~nm}$.

Fig. 1 depicts the optical components layout. The laser beam was firstly expanded using a tailored beam expander composed of 4 convergent lenses. The total magnification of the beam expander is equal to 11.67. This magnification produces a laser output beam with the optical characteristics described in Table 1.

Table 1

Laser optical characteristics after beam expansion.

\begin{tabular}{lrrc}
\hline & \multicolumn{1}{c}{$L_{532}$} & \multicolumn{1}{c}{$L_{635}$} & \multicolumn{1}{c}{$L_{850}$} \\
\hline Expanded diameter $(\mathrm{mm})$ & 40.9 & 12.8 & $46.6 \times 23.3$ \\
Illumination $\left(\mathrm{mm}^{2}\right)$ & 1313.8 & 128.7 & 852.8 \\
Optical power $(\mathrm{mW})$ & 4.5 & 4.9 & 3.0 \\
Irradiance $\left(\mathrm{W} / \mathrm{m}^{2}\right)$ & 3.4 & 38.1 & 3.5 \\
\hline
\end{tabular}

The three irradiances are under the maximum permissible exposure (MPE) in skin for large exposure times ( $10 \mathrm{~s}$ to $30 \mathrm{ks}$ ) which are $2000 \mathrm{~W} / \mathrm{m}^{2}$ for the $L_{635}$ and $L_{532}$ and $3990 \mathrm{~W} / \mathrm{m}^{2}$ for the $L_{850}$ [24]. All the experiments have been conducted with the respective eye protection for class $3 \mathrm{R}\left(L_{635}\right.$ and $\left.L_{532}\right)$ and class $3 \mathrm{~B}\left(L_{850}\right)$ lasers.

A layered target composed of several white translucent silicone membranes has been used with the purpose of studying the behavior of speckle patterns when a diffuse surface is moving. The target has been constructed by using 4 overlapped membranes with an approximated total thickness of $2 \mathrm{~mm}$. The target size was $30 \mathrm{~mm} \times 60 \mathrm{~mm}(\mathrm{~W} \times \mathrm{H})$. This target was connected to a piezoelectric actuator (PZA) (Physik Instrumente GmbH P-287), driven by a high voltage source (HVS) (Physik Instrumente GmbH, E-580) that is fed with a voltage signal generator (VSG) (Agilent 33220A). The displacement of the actuator and thus the membrane displacement are given by Eq. (1) [13] (Fig. 1):

$D=\frac{7000}{75} \times V(\mu \mathrm{m})$

where $D$ is the displacement in $\mu \mathrm{m}$ and $V$ is the electric potential in Volts applied by the signal generator to the high voltage amplifier. The laser was aligned to the target center to ensure that all the light interacts with the membrane.

A monochrome digital video camera (VC) (PixeLink - B741U) connected to a C-mount lens (Edmund 67715) has been used to record the speckle patterns. This model is widely used in laser speckle applications $[25,21]$. The maximum camera resolution was used to perform all the bench acquisitions $(1280 \times 1024$ pixels $)$ with an exposure time of $15 \mathrm{~ms}$ and a frame rate of 15 frames per second (fps) which was the maximum frame rate admissible by the VC with these parameters. The VC gain was fixed $(0 \mathrm{~dB})$ during the experiment in order to maintain a constant level of noise.

The speckle size was controlled by the lens aperture (f-number). Higher f-number corresponds to larger speckles [26] which means that this parameter requires a fine tuning in order to maintain enough light collection and a speckle size greater than or equal to the size of the VC pixels. In our experiment, the minimum speckle size was approximately 4 pixels/speckle. This speckle size ensures a correct spatial sampling because it meets the Nyquist limit for both $(x$ and $y)$ spatial dimensions. Since the spatial resolution is not a key factor in this experiment, the reduction of the spatial resolution that comes from the use of a large speckle size is not an issue.

Data acquisition has been performed using a software interface developed in Python 2.7 (32 bits) with the functions provided by the opencv library, version 2.4.7 [27]. Processing algorithm have 
Table 2

Maximum membrane velocities according to the excitation signal parameters amplitude and frequency. The velocities are expressed in millimeters per second.

\begin{tabular}{llllll}
\hline Amplitude $\left(V_{p p}\right)$ & Displacement $(\mathrm{mm})$ & \multicolumn{5}{l}{ Frequency $(\mathrm{Hz})$} \\
\cline { 3 - 6 } & & 1 & 2 & 3 & 5 \\
\hline 0.5 & 0.05 & 0.15 & 0.29 & 0.44 & 0.60 \\
1 & 0.09 & 0.29 & 0.59 & 0.88 & 1.47 \\
2 & 0.19 & 0.59 & 1.17 & 1.76 & 2.93 \\
4 & 0.37 & 1.17 & 2.34 & 3.52 & 5.86 \\
\hline
\end{tabular}

been developed in Matlab ${ }^{\circledR}$. This test set up was used to perform two distinct trials.

\subsection{Vibration frequency detection}

The first test consists in recording the speckle pattern changes during a continuous movement of the membrane in order to identify its vibration frequency. The target was actuated with a sinusoidal signal with different amplitudes and frequencies. A video sequence of $7 \mathrm{~s}$ was recorded for each sinusoidal signal. The same conditions and the same alignment were used during all the acquisitions.

A total of four distinct values of amplitudes and frequencies were used. These values and the maximum membrane velocities [13] are detailed in Table 2.

Data analysis was performed with the aim to extract the oscillation frequency of the silicone membrane. This analysis was applied in a group of pixels of the image, i.e., it was performed in preselected pixel windows. The power spectral density (PSD) was computed in every single pixel of the analysis window and the mean of PSD functions was associated with this window.

The PSD is defined as the modulus of the square Fourier transform of the signal:

$\operatorname{PSD}_{(x, y)}\left(f_{t}\right)=\left|\sum_{k=1}^{K} A(x, y, k) \times e^{\frac{-i 2 \pi(k-1)\left(f_{t}-1\right)}{K}}\right|^{2}$,

where $f_{t}$ is the frequency present in the signal, $K$ the total number of frames of the video, $A$ is the three-dimensional structure composed by all images, and $x$ and $y$ represent a pixel position (line, column). In fact, the temporal evolution of the pixel $(x, y)$ intensity is the raw signal input of the PSD and can be represented as $I_{x, y}[t]$.

Before applying the PSD to the pixel intensity function $\left(I_{x, y}[t]\right)$ a filtering stage was applied to remove the signal DC frequency. A 4 th order Butterworth filter with a cut-off frequency of $0.1 \mathrm{~Hz}$ was used to perform this operation.

For each acquisition, analysis windows of width 3, 9, 17 and 81 pixels were used (the red squares highlighted in Fig. 2). The dashed red squares are examples of 81 points windows. Hereinafter, and for convenience, these windows were numbered from up to bottom and left to right, causing the upper left corner window to be named as $W 1$, the bottom left corner to be named as $W 3$ and the bottom right corner window to be named as $W 9$.

According to these parameters, and since all the combinations of windows size and position were analyzed, 36 PSD functions were computed for each acquisition. For each one of the PSD, the power and frequency of the most intense peak were stored and used to evaluate this method.

\subsection{Velocity profile detection}

The second bench-test performed aimed at evaluating LS data when the membrane is moving during a small period (single sinusoidal period) while the acquisition is in progress. The aim of this test was to evaluate the performance of the 3 wavelengths for the velocity profile reconstruction. The same alignment conditions were maintained during this test.

In this test, 4 different periods were used to feed the piezoelectric actuator: $1 \mathrm{~s} ; 2 \mathrm{~s} ; 3 \mathrm{~s}$; and $5 \mathrm{~s}$. In addition, 3 distinct amplitudes, $2 V_{p p}, 4 V_{p p}$ and $8 V_{p p}$ were used, leading to a total of 12 acquisitions. Once again, videos with $7 \mathrm{~s}$ length were acquired for each movement in order to ensure the complete record of the movement with the largest period ( $5 \mathrm{~s}$ ).

The image was restricted to its inner area (green rectangle in Fig. 2) because it is expected that the most relevant information is represented in the center of the speckle pattern. Another trivial advantage of this approach was the computational time reduction. The green rectangle corresponds to an area of $427 \times 341$ pixels.

The algorithm used to extract the velocity profile information was based on a two-dimensional correlation coefficient computation between two consecutive speckle patterns. This approach has already been used, for a different application [28], with timevariant LS data. The correlation coefficient $(r)$ is determined as:

$r=\frac{\sum_{x} \sum_{y}\left(A_{x y}-\bar{A}\right)\left(B_{x y}-\bar{B}\right)}{\sqrt{\left(\sum_{x} \sum_{y}\left(A_{x y}-\bar{A}\right)^{2}\right)\left(\sum_{x} \sum_{y}\left(B_{x y}-\bar{B}\right)^{2}\right)}}$

where $A$ and $B$ are two consecutive images, $\bar{A}$ and $\bar{B}$ represent the average pixel intensity of each image and the indexes $x$ and $y$ the pixel position in the image. When this method is applied to a video with $K$ number of frames, by using a time sliding window with complete overlapping, $K-1$ coefficients are computed.

As the membrane moves faster, the $r$ value becomes smaller because the speckle pattern changes quickly. To easily evaluate the quality of the estimation, the $r$ value was normalized between 0 and $1\left(r^{\prime}\right)$ and inverted $\left(1-r^{\prime}\right)$ to better match the absolute value of the real velocity.

\subsection{In vivo test}

The in vivo tests were performed in order to evidence the distinct tissue interaction of each wavelength. The test consisted in evaluating the movement of the radial artery of two distinct subjects by using the instrumentation and the principles described above. The radial artery LS data was recorded simultaneously with a photoplethysmogram (PPG) acquired with a pulse oximeter at the index finger of the left hand. The two subjects provided written, informed consent prior to participation and the study was carried out in accordance with the Declaration of Helsinki.

The PPG was recorded with a custom made probe and front end circuit [29] that contains a red and infrared LED but only the red channel was used. The electrical signal was then digitized with a National Instruments data acquisition device (NI-DAQ 6210) connect to the host PC.

Fig. 3 shows the assemblage used to acquire signals during the in vivo trials. The laser support (C) and beam expander (B) were mounted in a horizontal position and the light was directed to the wrist using a mirror (D). The digital VC (A) was placed at a distant position from the radial artery using a laboratory clamp. Finally, the PPG probe (E) was placed in the index finger. The arm of the volunteer was kept fixed in a position during the data acquisition procedure. Two healthy subjects, a male ( 24 years) and a female (27 years) were submitted to this test that was performed with the volunteers in the seated position.

It is evident that the physiological system is a more complex system than the bench-test presented above and, for this reason, a higher sampling frequency for the video signal was required. In this case, a sampling frequency of $50 \mathrm{fps}$ was used with images of resolution $320 \times 240$ pixels. Three acquisitions have been performed on each subject and for each light source. 


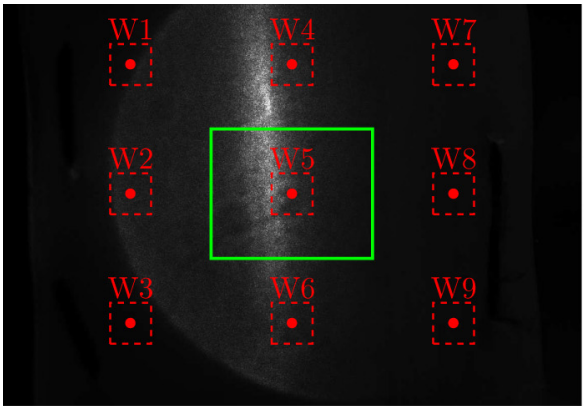

(a)

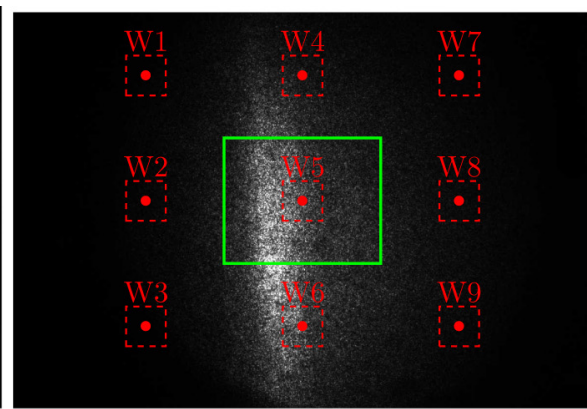

(b)

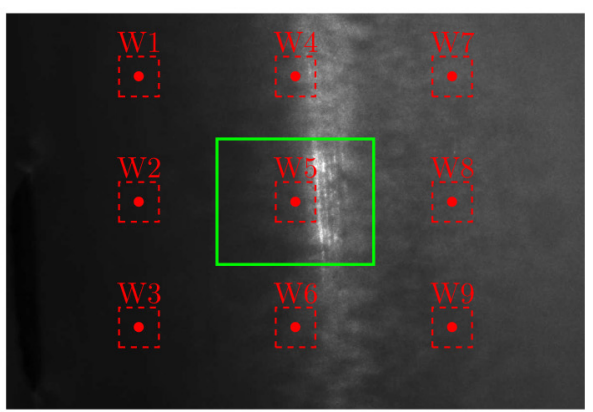

(c)

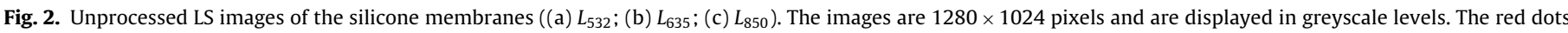

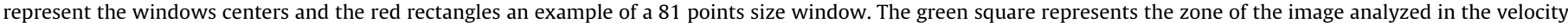

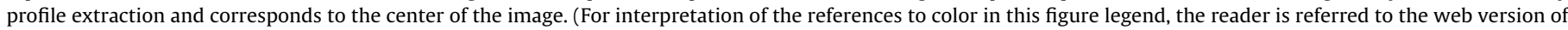
this article.)

The PSD-based algorithm was not applied to this dataset because the results obtained for the correlation coefficient algorithm were able to estimate both the artery vibration frequency (heart rate) and the arterial pulse waveform profile. The correlation algorithm, used in Section 2.3, was applied to these data without image restriction due to the image lower resolution.

Two different pre-processing image techniques, along with a final data filtering stage, were used in order to enhance the final result. The first pre-processing technique consisted in an image binarization by using an adaptive threshold which corresponds to the midpoint of the intensity range presented in the image. The pixels with intensities higher than the threshold were considered as ' 1 ' and the pixels with lower value were considered as ' 0 '.

The second pre-processing method consisted in the histogram equalization. To perform the equalization, the Matlab ${ }^{\circledR}$ function histeq from the Image Processing Toolbox was used. The minimization criterion, used by this function, is based on contrast enhancement [30].

\section{Results and discussion}

The current section presents the results obtained for both bench-test and in vivo test. Fig. 2 shows three LS images acquired from the bench-test. Since the penetration of infra-red light is higher than the red and green light (in the silicone membrane and skin) [31], the speckles appear blurred even when the membrane is not moving (Fig. 2(c)). By observing this figure, it appears that the speckle pattern formed with the $L_{850}$ comes from an internal silicone membrane whereas the $L_{532}$ and the $L_{635}$ patterns come

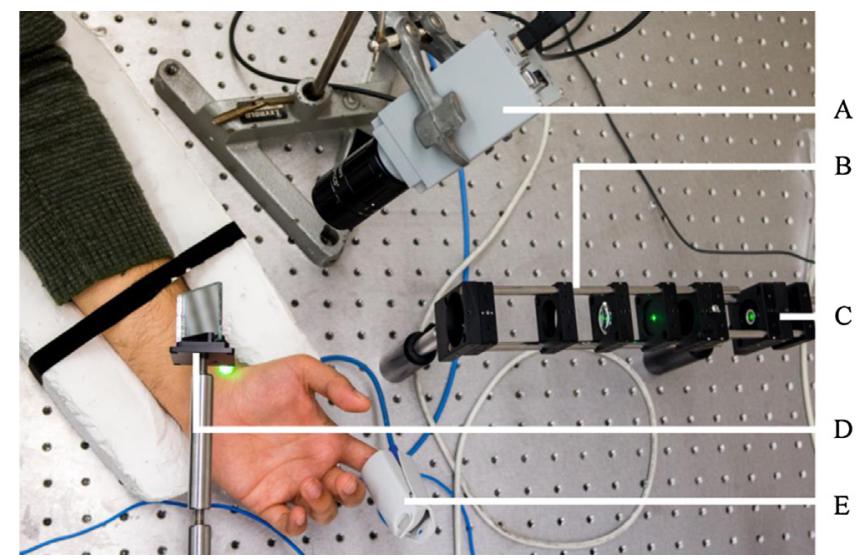

Fig. 3. Photography of the test performed in vivo. (A) Digital Video Camera; (B) Beam expander; (C) Laser support; (D) Mirror; (E) PPG probe. from the superficial layer. This can be a possible cause for the blurring effect visible in the $L_{850}$ speckle pattern because of multiple scattering events.

\subsection{Vibration frequency detection}

The periodic movement of the target was evaluated as the root mean square error $\left(\epsilon_{r m s}\right)$ between the true membrane oscillation

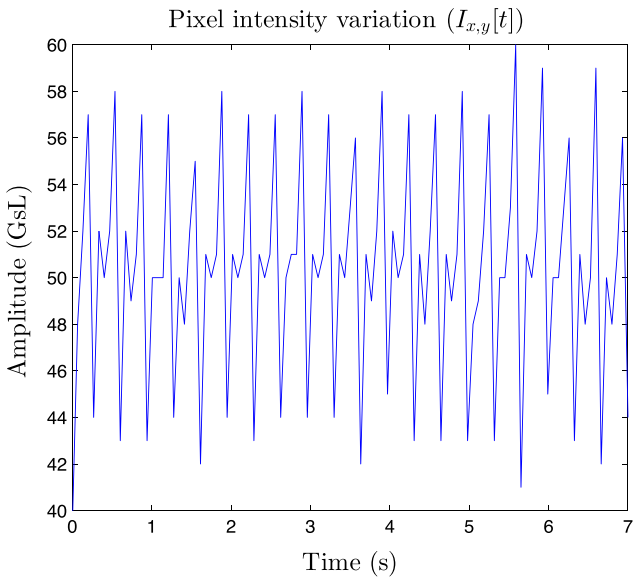

(a)

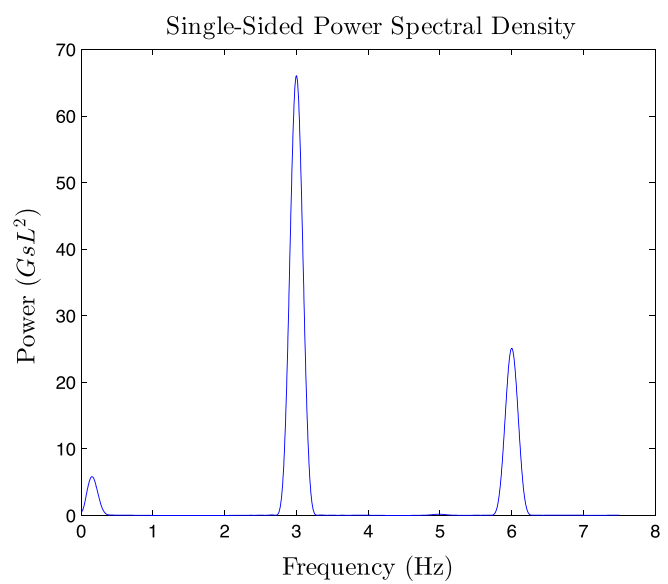

(b)

Fig. 4. (a) Temporal variation of the central pixel intensity of the acquisition characterized by an amplitude of $1 V_{p p}$ and frequency of oscillation of $3 \mathrm{~Hz}$. The amplitude is represented in greyscale levels (GsL). (b) Power spectral density (PSD) computed in the window W5 with 81 pixels size. 


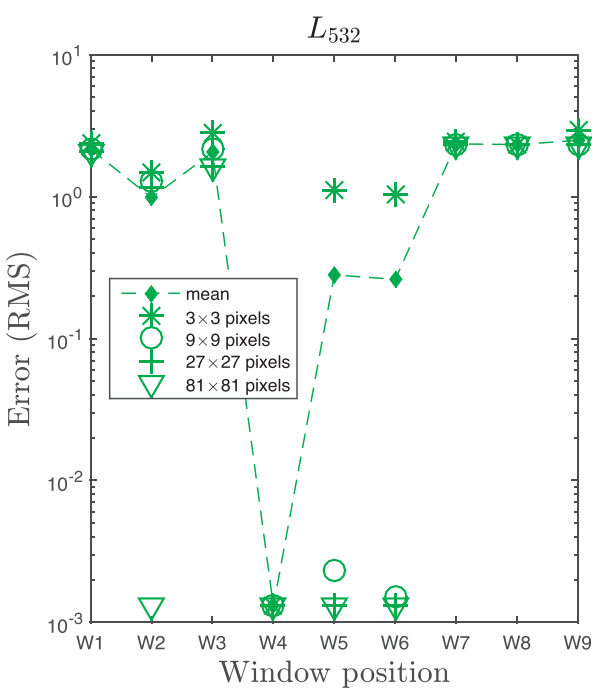

(a) Green laser

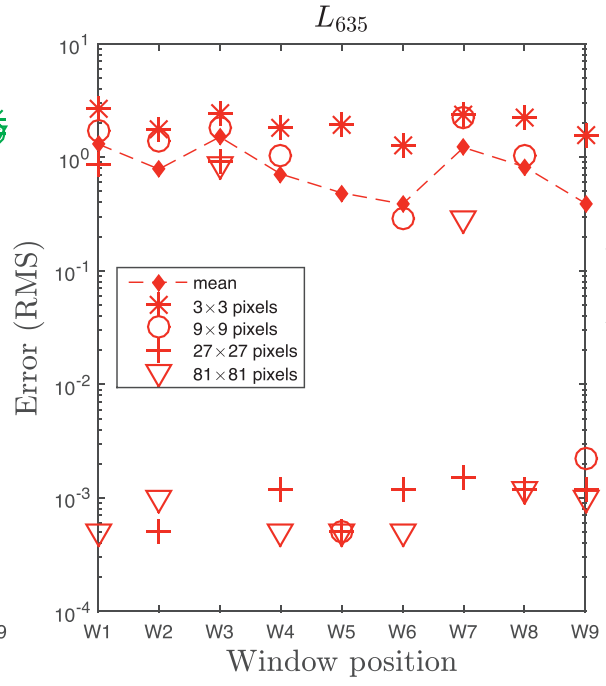

(b) Red laser

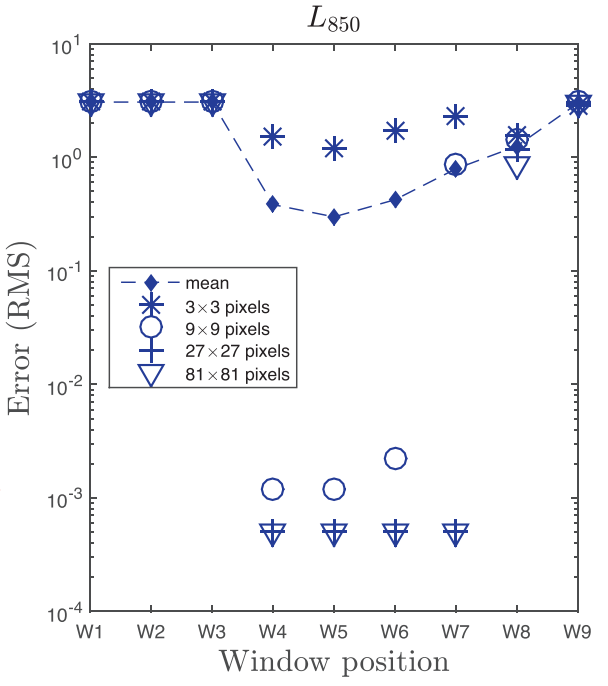

(c) Infra-red laser

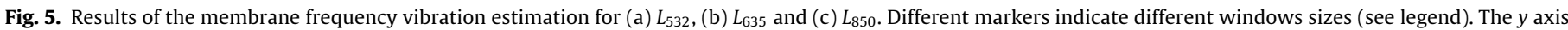
is presented in log scale.

frequency and the estimated oscillation frequency. Fig. 4(a) represents the temporal evolution of the central pixel $\left(I_{x, y}[t]\right)$ of the W5 in a LS data. The mean PSD computed using the window W5 with size 81 pixels is shown in Fig. 4(b). This PSD corresponds to the analysis performed in the acquisition with membrane movement with an amplitude of $1 V_{p p}$ and with a target vibration frequency of $3 \mathrm{~Hz}$.

The most powerful frequency occurs at $3 \mathrm{~Hz}$ which is expected due to the applied movement. A second peak with high intensity is also visible at $6 \mathrm{~Hz}$ and corresponds to the second harmonic. The high power of the harmonics can be explained by the intermediate fluctuations of the speckle pattern within a periodic movement. During a cycle, the speckle pattern suffers from many variations which cause secondary frequencies, different from the vibration frequency. These secondary frequencies are visible in the PSD and can lead to some misidentification of the real oscillation frequency. This phenomenon occurs when the speckle observation is made in a position that is non-normal to the surface leading to a pseudorandom speckle noise [2]. Moreover, the silicone membrane is composed of many layers which can present small independent oscillation leading to secondary frequencies.

The $\epsilon_{r m s}$ is computed for each one of the possible combinations of the two degrees of freedom (window position and window size) for all the acquisitions. This means that all the signals, regardless of the amplitude and frequency, are evaluated at the same time. Eq. (4) represents the computation of the error where $N$ is the total number of files (12) and $f$ the frequency in Hz. The letters $s$ and $p$ represent a specified size and window position, respectively.

$\epsilon_{r m s}^{s, p}=\sqrt{\frac{\sum_{n=1}^{N}\left(f_{n, s, p}^{\text {estimated }}-f_{n, s, p}^{\text {true }}\right)^{2}}{N} .}$

The results are presented in Fig. 5 . The $L_{532}$ case shows lower errors in the central column windows (W4, W5 and W6), independently of the window size. With a minimum error of $10^{-2.9}$ $(1.3 \mathrm{mHz})$, the green laser represents the poorest result. The speckle images of Fig. 2 show the reason for the best results of the central column windows. The membrane curvature causes an anisotropic light diffusion, mainly in the green wavelength, causing the areas along the central column to present higher emittance. Outside these areas, the image quality, in terms of LS information, is weaker.
In the case of the $L_{635}$, the results evidence that the window size is significantly more important than its actual position. By analyzing the results obtained for the largest windows (81 pixels) the oscillation frequency is identified, in almost all the cases, with an error close to $10^{-3.3}(0.5 \mathrm{mHz})$. This value, in terms of the analyzed frequency range $(1-3 \mathrm{~Hz})$, represents an error between $0.02 \%$ and $0.05 \%$.

For the $L_{850}$ case, Fig. 5(c), the position of the window and its size are relevant parameters for a good vibration frequency identification. The minimum error obtained with this light source is $0.5 \mathrm{mHz}$ which is the same as the $L_{635}$ case. This value happens to be related with the FFT resolution. Both the $L_{635}$ and $L_{850}$ achieved similar performances on the identification of the vibration frequency, showing a more isotropic diffraction of the light inside the silicone membrane (see the effect of the window position).

\subsection{Velocity profile detection}

To evaluate the results for velocity profile estimation the absolute value of the membrane velocity was normalized in order to be

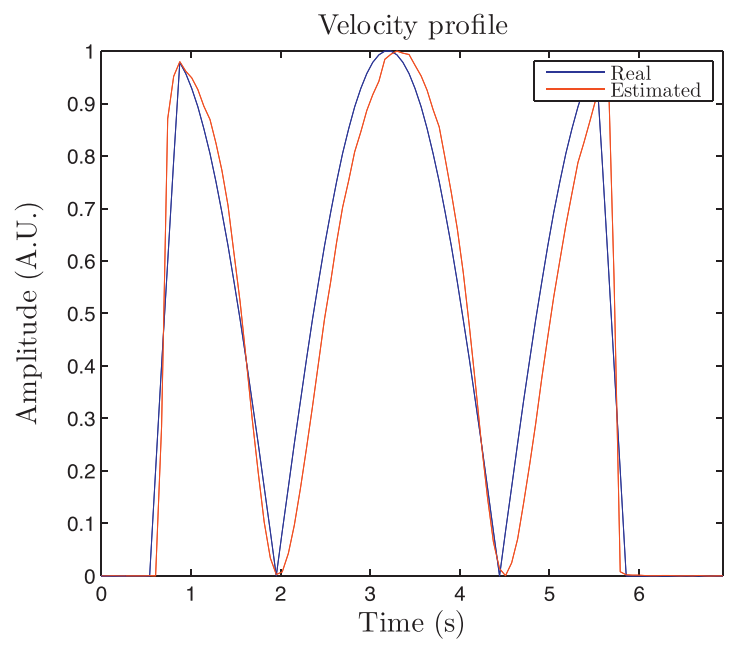

Fig. 6. Plot of $1-r^{\prime}$ along time (red line) and absolute velocity of the target (blue line) of the actuator signal with amplitude of $2 V_{p p}$ and $5 \mathrm{~s}$ of period. (For interpretation of the references to color in this figure legend, the reader is referred to the web version of this article.) 
Table 3

Results of the RMS error of the velocity profile estimation with $L_{532}, L_{635}$ and $L_{850}$ data. Values presented in percentage.

\begin{tabular}{rllrr}
\hline & & & \multicolumn{3}{l}{ Deriod $(\mathrm{s})$} & & \\
\cline { 3 - 4 } & & 0.19 & 0.37 & 0.75 \\
\hline \multirow{4}{*}{$L_{532}$} & 1 & 20.68 & 11.87 & 19.31 \\
& 2 & 18.63 & 15.08 & 12.68 \\
& 3 & 15.55 & 9.92 & 9.52 \\
& 5 & 12.22 & 9.45 & 10.21 \\
& 1 & 17.38 & 18.96 & 19.58 \\
$L_{635}$ & 2 & 20.83 & 21.68 & 24.91 \\
& 3 & 12.54 & 20.66 & 25.11 \\
& 5 & 10.31 & 16.78 & 26.64 \\
& 1 & 16.27 & 14.78 & 13.26 \\
$L_{850}$ & 2 & 16.11 & 12.19 & 13.83 \\
& 3 & 20.42 & 13.97 & 9.02 \\
& 5 & 21.62 & 22.64 & 14.89 \\
\hline
\end{tabular}

compared for both profiles. Fig. 6 shows an example of a real and estimated velocity profile. The real profile (blue line) is the normalized absolute value of the derivative of the voltage signal produced by the VSG. The red line is the inverted and normalized correlation coefficient $\left(1-r^{\prime}\right)$.

The root mean square error was computed between the actual velocity absolute value and the profile extracted by this algorithm. The results are presented in Table 3 and Fig. 6, and they show how the different factors, wavelength, period and displacement, influence the error of the reconstruction.

As it can be seen, in the $L_{532}$ case, a clear tendency is observed where movements with larger periods show better results. The mean errors for each period were respectively $17 \%, 16 \%, 12 \%$ and $11 \%$. The lower error was achieved for the movement with $0.75 \mathrm{~mm}$ of displacement and $5 \mathrm{~s}$ period with a value of $9.5 \%$. These results are easily explained because both large periods and small displacement correspond to lower velocities. In fact, the low frame rate of the acquisitions ( $15 \mathrm{fps}$ ) is a major obstacle when rapid movements occur. Moreover, the high compliance of the membrane causes it to continue to move even after the piezoelectric actuator has stopped. This effect is more relevant in the rapid movements because the target shows higher linear momentum and takes more time to stops completely.

In the case of the $L_{635}$, the error decreases for movements with large periods and small displacements. The minor error is $10.3 \%$ which occurs for the largest period ( $5 \mathrm{~s}$ ) and the smallest displacement $(0.19 \mathrm{~mm})$ (the lowest velocity) which is the case presented in Fig. 6. The mean errors for each period were respectively $19 \%$, $22 \%, 19 \%$ and $18 \%$.

Lastly, for the case of the $L_{850}$, the results present a different situation. In this case, the tendency is that movements with large displacement have smaller error. Nevertheless the results show poor correlation with the displacement and period parameters. For example, the mean errors for each period were $15 \%, 14 \%, 14 \%$ and $21 \%$ respectively.

Comparing the 3 wavelengths, the mean errors were $13.8 \% 19.6$ $\%$ and $15.8 \%$ for $L_{532}, L_{635}$ and $L_{850}$ respectively. The anisotropic reflection of the $L_{532}$ cause most of the light to be reflected with the region of interest leading to a highest signal-to-noise ratio.

\subsection{In vivo test}

The in vivo application presented a much lower signal-to-noise ratio (SNR) when compared to the bench-test application. The $L_{850}$ data was considered unusable for the application of the present algorithms due to its higher tissue penetration [32]. Higher penetrations cause more scattering events, leading to an output beam

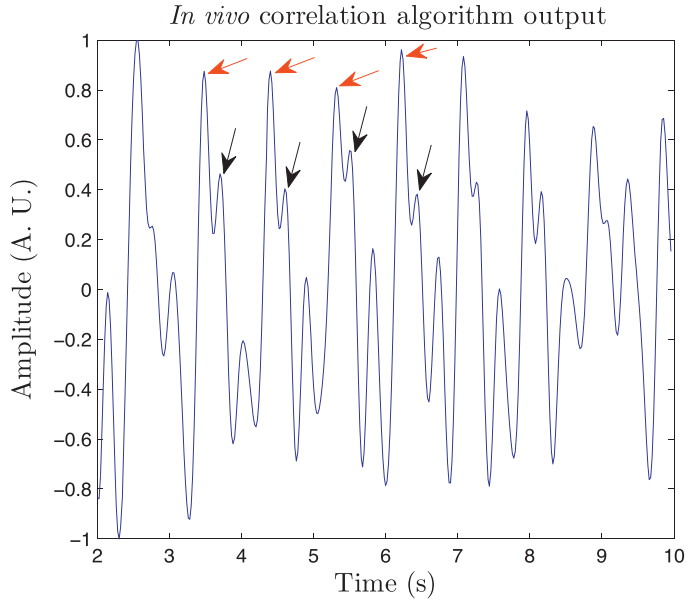

(a)

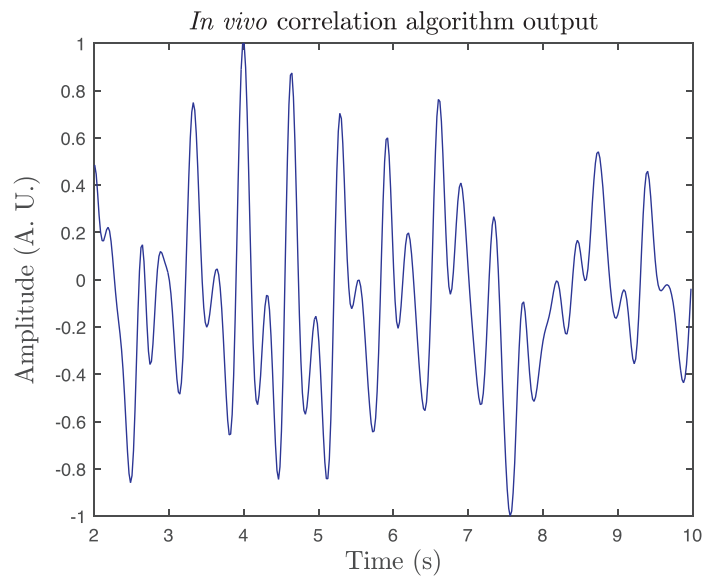

(b')

Fig. 7. Output signal of the correlation algorithm (including the final filtering) to the in vivo test S4 (a) and S10 (b). Red arrows show the probable systolic peak and black arrows show the probable dicrotic notch. (For interpretation of the references to color in this figure legend, the reader is referred to the web version of this article.)

less coherent. The coherence reduction limits the laser speckle effect and reduces the speckle pattern contrast.

The image correlation algorithm was used in order to extract the heart rate (HR) and pulse distension profile. The resultant signal was further filtered with a 8th order Butterworth filter with a band-pass between $0.3 \mathrm{~Hz}$ and $4 \mathrm{~Hz}$ (this band corresponds to HRs between 18 and $240 \mathrm{bpm}$ which is an acceptable physiological range). This frequency-domain filtering is also used to remove tremors which can occur during the acquisitions.

The laser speckle data were processed offline due to VC interface constraints. However, the processing time, using the developed Matlab ${ }^{\circledR}$ algorithm in a laptop (Core i7 - M620 @ $2.67 \mathrm{GHz}$ ) was of $\approx 1.5 \mathrm{~ms}$ per correlation coefficient. The processing time is much less than the exposure time ( $15 \mathrm{~ms})$, which is the minimum time to acquire an image. An online processing method could be implemented in future with simple interface modifications.

An example of the extracted signal, after all the processing stages is presented in Fig. 7. This image corresponds to the processing of the data S4 and S10 represented in Table 4 . This image shows the pulse pressure waveform extracted with the correlation coefficient after filtering and normalization for both wavelengths. The arrows indicate pulse waveform feature points with clinical relevance [17] like systolic peak and dicrotic notch.

As stated earlier, 6 signals were acquired for each subject ( 3 for the $L_{532}$ source and 3 for the $L_{635}$ source). The results of the 
Table 4

Results of heart rate (HR) estimation with in vivo conditions. The values in the table are expressed in beats/minute (bpm).

\begin{tabular}{|c|c|c|c|c|c|c|c|c|}
\hline & Data set & $\mathrm{S} 1$ & $\mathrm{~S} 2$ & $S 3^{\mathrm{a}}$ & S4 & S5 & S6 & $p \epsilon_{r m s}$ \\
\hline \multirow{6}{*}{$L_{532}$} & Effective HR & 62.3 & 62.3 & 64.1 & 65.9 & 67.8 & 67.8 & \\
\hline & No pre-process & 61.4 & 62.4 & 69.6 & 65.7 & 67.6 & 67.2 & $2.28(0.50)$ \\
\hline & Binarization & 61.5 & 61.9 & 69.5 & 65.7 & 67.4 & 67.4 & $2.24(0.48)$ \\
\hline & Hist. equal. & 61.4 & 62.3 & 69.6 & 65.7 & 67.6 & 67.2 & $2.28(0.50)$ \\
\hline & $d \epsilon_{r m s}$ & 0.79 & 0.22 & 5.46 & 0.18 & 0.26 & 0.50 & \\
\hline & Data set & S7 & S8 & S9 & S10 & S11 & $\mathrm{S} 12$ & $p \epsilon_{r m s}$ \\
\hline \multirow{5}{*}{$L_{635}$} & Effective HR & 64.1 & 65.9 & 67.7 & 82.4 & 89.7 & 60.4 & \\
\hline & No pre-process & 66.4 & 66.0 & 68.7 & 82.4 & 88.6 & 59.6 & 1.15 \\
\hline & Binarization & 66.7 & 65.6 & 68.7 & 82.5 & 88.5 & 59.6 & 1.26 \\
\hline & Hist. equal. & 66.4 & 65.9 & 68.7 & 82.4 & 88.6 & 59.6 & 1.15 \\
\hline & $d \epsilon_{r m s}$ & 2.38 & 0.22 & 0.92 & 0.05 & 1.13 & 0.82 & \\
\hline
\end{tabular}

a Data set tainted by artifacts.

HR identification, for each one of the pre-processing techniques and for the 12 data sets, are shown in Table 4 . To analyze these numbers, the root mean square error $\left(\epsilon_{r m s}\right)$ was computed for each pre-processing technique and for each acquired data set. These two $\epsilon_{r m s}$ are presented both in Table 4 with the letters $p \epsilon_{r m s}$, error computed with all the data sets for each pre-processing technique (within parenthesis are the results excluding S3), and $d \epsilon_{r m s}$, error computed with all the pre-processing technique for a given data set.

These results show that both the pre-processing techniques (binarization and histogram equalization) and the absence of preprocessing achieved similar results for both coherent light sources. These fact can indicate that, since speckle information is encoded in decorrelation and contrast [33], the speckle data is unresponsive to these techniques.

The $p \epsilon_{r m s}$ for the $L_{532}$ varied between $2.24 \mathrm{bpm}$ and $2.28 \mathrm{bpm}$. If we look closer to the $d \epsilon_{r m s}$ of the $S 3$, it was clearly affected by movement artifacts. This conclusion is supported by the value of $5.46 \mathrm{bpm}$ which is much higher than the other cases and statistically consists in an outlier. When this data-set is excluded from the $p \epsilon_{\text {rms }}$ computation, its values drop for $0.50,0.48$ and 0.50 .

By analyzing the results of the $L_{635}$ it can be seen that it achieved the best result in the $\mathrm{S} 10$ data set with an error of $0.05 \mathrm{bpm}$ in the HR identification. On the other hand, if we look globally to the $p \epsilon_{r m s}$ the results are better than the first light source but only if the S3 is not excluded. When the $\mathrm{S} 3$ is excluded from the analysis, the $p \epsilon_{r m s}$ of the $L_{532}$ become much better (approximately half of the error) than the $L_{635}$.

Observing Fig. 7, which represents the best cases for both wavelengths, it can be concluded that the signal presented for the $L_{532}$ shows a steadier behavior. The arterial waveform dicrotic notch is also easier to identify in the signal extracted with this wavelength.

\section{Conclusions}

The present work describes the study conducted to evaluate and quantify the effect of different coherent light sources in the evaluation of the arterial pulse waveform using laser speckle analysis techniques. The test performed in vivo showed that different light wavelengths have distinct abilities to extract the arterial pulse waveform.

The combination of the proposed instrumentation and algorithms were able to identify the vibration frequency of a silicone membrane phantom. The $L_{635}$ and $L_{850}$ light sources achieved the best results with a minimum identification error of $0.5 \mathrm{mHz}$ in the vibration frequency. Although the minimum error of the $L_{532}$ light source was about $1.3 \mathrm{mHz}$, the results were close to each other. In all cases, the largest windows ( 81 and 27 pixels) achieved the best performances. Another important conclusion of this study was the fact that the $L_{532}$ light was anisotropically scattered in the silicone membranes.

The profile detection study aimed to simulate the movement of a biological tissue, like the skin of the wrist, when the pulse pressure wave travels in the radial artery. The estimation of the absolute value of the velocity profile was achieved with a mean error of $13.8 \%$ for the $L_{532}, 19.6 \%$ for the $L_{635}$ and $15.8 \%$ for the $L_{850}$. In this case, the $L_{532}$ achieved the best results, which is probably due to its lower phantom penetration.

In the case of biological measurements several adaptations were made to improve the results. The most significant was the limitation of the size of the acquired images in order to increase the sampling frequency. With a frequency rate of $50 \mathrm{~Hz}, 12$ distinct data-sets were acquired and processed with an algorithm based on the image correlation used in the bench-tests. The two pre-processing techniques that were applied (binarization and histogram equalization) proved to be ineffective. The $L_{532}$ achieved the best results if we exclude the $\mathrm{S} 3$ data-set, which was most likely corrupted by motion artifacts. On the other hand the $L_{850}$ was unable to properly record the arterial pulse waveform which indicates that wavelengths in the near infra-red are not suitable for this type of assessment.

The shorter wavelength laser source $\left(L_{532}\right)$ showed better consistency in extracting the arterial pulse waveform. The lowest tissue penetration of the green light improves the signal-to-noise ratio when the laser speckle is used for skin vibration measurements. These results are promising for a future application of a system that combines blood flow measurement (red light) with artery distension information (green light) using multiple-wavelength light sources. However, more in vivo test, with more volunteers, are required to show the influence of the multi-spectral system. For example, obese individuals must be analyzed to test the infra-red light wavelengths for the case of deeper arteries and thick fat layers.

\section{Conflict of interest}

The authors state that they have no conflict of interest to declare.

\section{Acknowledgement}

The authors acknowledge the support from Fundação para a Ciência e Tecnologia (FCT) for funding a doctoral scholarship (SFRH/BD/89585/2012).

\section{References}

[1] H. Hirabayashi, T. Matsuo, H. Ishizawa, H. Kanai, T. Nishimatsu, Surface roughness evaluation by laser speckle, in: International Joint Conference SICE-ICASE, 2006, IEEE, 2006, pp. 5809-5812.

[2] P. Martin, S. Rothberg, Introducing speckle noise maps for laser vibrometry, Opt. Lasers Eng. 47 (3) (2009) 431-442. 
[3] L.M. Novak, M.C. Burl, Optimal speckle reduction in polarimetric sar imagery, IEEE Trans. Aerosp. Electr. Syst. 26 (2) (1990) 293-305.

[4] X. Zong, A.F. Laine, E.A. Geiser, Speckle reduction and contrast enhancement of echocardiograms via multiscale nonlinear processing, IEEE Trans. Med. Imaging 17 (4) (1998) 532-540.

[5] M. Bashkansky, J. Reintjes, Statistics and reduction of speckle in optical coherence tomography, Opt. Lett. 25 (8) (2000) 545-547.

[6] J.D. Briers, S. Webster, Laser speckle contrast analysis (lasca): a nonscanning, full-field technique for monitoring capillary blood flow, J. Biomed. Opt. 1 (2) (1996) 174-179.

[7] A. Humeau-Heurtier, E. Guerreschi, P. Abraham, G. Mahe, et al., Relevance of laser Doppler and laser speckle techniques for assessing vascular function: state of the art and future trends, IEEE Trans. Biomed. Eng. 60 (3) (2013) 659-666.

[8] V.V. Tuchin, Coherent optical techniques for the analysis of tissue structure and dynamics, J. Biomed. Opt. 4 (1) (1999) 106-124.

[9] P. Vaz, P. Santos, E. Figueiras, C. Correia, A. Humeau-Heurtier, J. Cardoso, Laser speckle contrast analysis for pulse waveform extraction, in: Nov. Biophotonics Tech. Appl. III, vol. 9540, SPIE, Munich, 2015, pp. 954006-954007.

[10] T. Matsuo, H. Hirabayashi, H. Ishizawa, H. Kanai, T. Nishimatsu, Application of laser speckle method to water flow measurement in plant body, in: International Joint Conference SICE-ICASE, 2006, IEEE, 2006, pp. 3563-3566.

[11] A. Veber, A. Lyashedko, E. Sholokhov, A. Trikshev, A. Kurkov, Y. Pyrkov, A. Veber, V. Seregin, V. Tsvetkov, Laser vibrometry based on analysis of the speckle pattern from a remote object, Appl. Phys. B: Lasers Opt. 105 (3) (2011) 613-617.

[12] C. Joenathan, B. Franze, P. Haible, H. Tiziani, Speckle interferometry with temporal phase evaluation for measuring large-object deformation, Appl. Opt. 37 (13) (1998) 2608-2614.

[13] P. Vaz, D. Capela, T. Pereira, C. Correia, R. Ferreira, A. Humeau-Heurtier, J. Cardoso, Use of laser speckle and entropy computation to segment images of diffuse objects with longitudinal motion, in: Second International Conference on Applications of Optics and Photonics $(92861 \mathrm{H})$, vol. 9286 of Proceedings SPIE, 2014, http://dx.doi.org/10.1117/12.2060845.

[14] G. Ramachandran, M. Singh, Three-dimensional reconstruction of cardiac displacement patterns on the chest wall during the p, qrs and t-segments of the ecg by laser speckle inteferometry, Med. Biol. Eng. Comput. 27 (5) (1989) 525-530.

[15] T. Asakura, N. Takai, Dynamic laser speckles and their application to velocity measurements of the diffuse object, Appl. Phys. 25 (3) (1981) 179-194.

[16] J. Ohtsubo, T. Asakura, Velocity measurement of a diffuse object by using timevarying speckles, Opt. Quantum Electr. 8 (6) (1976) 523-529.

[17] T. Pereira, I. Santos, T. Oliveira, P. Vaz, T. Correia, T. Pereira, H. Santos, H. Pereira, V. Almeida, J. Cardoso, et al., Characterization of optical system for hemodynamic multi-parameter assessment, Cardiovasc. Eng. Technol. 4 (1) (2013) 87-97.
[18] D. Briers, D.D. Duncan, E. Hirst, S.J. Kirkpatrick, M. Larsson, W. Steenbergen, T. Stromberg, O.B. Thompson, Laser speckle contrast imaging: theoretical and practical limitations, J. Biomed. Opt. 18 (6) (2013) 066018.

[19] M. Bahrawi, N. Farid, M. Abdel-Hady, Speckle cross-correlation method in measuring fine surface displacements, J. Atomic Mol. Opt. Phys. (2012).

[20] Y. Beiderman, I. Horovitz, N. Burshtein, M. Teicher, J. Garcia, V. Mico, Z. Zalevsky, Remote estimation of blood pulse pressure via temporal tracking of reflected secondary speckles pattern, J. Biomed. Opt. 15 (6) (2010) 061707, http://dx.doi. org/10.1117/1.3505008.

[21] Z. Zalevsky, Y. Beiderman, I. Margalit, S. Gingold, M. Teicher, V. Mico, J. Garcia, Simultaneous remote extraction of multiple speech sources and heart beats from secondary speckles pattern, Opt. Express 17 (24) (2009) 21566-21580.

[22] M. Nemati, C.N. Presura, H.P. Urbach, N. Bhattacharya, Dynamic light scattering from pulsatile flow in the presence of induced motion artifacts, Biomed. Opt. Express 5(7)(2014)2145-2156, http://dx.doi.org/10.1364/BOE.5.002145,URL: http://www.opticsinfobase.org/boe/abstract.cfm?URI=boe-5-7-2145.

[23] M. Nemati, L.G. Paroni, N. Bhattacharya, H.P. Urbach, Fluid pulsation detection in presence of induced motion artifacts using speckle techniques, AIP Conf. Proc. 1600 (2014) 215-222, http://dx.doi.org/10.1063/1.4879585.

[24] R. Matthes, Revision of guidelines on limits of exposure to laser radiation of wavelengths between $400 \mathrm{~nm}$ and 1.4 microm. International Commission on Non-Ionizing Radiation Protection, Health Phys. 79 (4) (2000) 431-440, URL: http://www.ncbi.nlm.nih.gov/pubmed/11007467.

[25] Z. Zelevsky, J. Garcia, Simultaneous remote monitoring of biomedical parameters via laser speckle patterns, Photon. Int. (2012) 18-20.

[26] S.S. Kazmi, L.M. Richards, C.J. Schrandt, M.A. Davis, A.K. Dunn, Expanding applications, accuracy, and interpretation of laser speckle contrast imaging of cerebral blood flow, J. Cereb. Blood Flow Metabol. 35 (2015) 1076-1084.

[27] I. Corporation, Open Source Computer Vision Library, 2014, URL: http://opencv. org/ (accessed 20.12.13).

[28] Y.N. Kulchin, O. Vitrik, A. Lantsov, N. Kraeva, Correlation method for processing speckle patterns of dynamic light scattering by small particles based on spatial averaging of data, Optoelectron. Instrum. Data Process. 46 (3) (2010) 282-286.

[29] P. Santos, Photoplethysmographic logger with contact force and hydrostatic pressure monitoring (Master's thesis), University of Coimbra, 2012.

[30] MATLAB, MathWorks Documentation Center, 2014, URL: http://www. mathworks.com/help/images/ref/histeq.html (accessed 23.10.14).

[31] K.R. Byrnes, R.W. Waynant, I.K. Ilev, X. Wu, L. Barna, K. Smith, R. Heckert, H. Gerst, J.J. Anders, Light promotes regeneration and functional recovery and alters the immune response after spinal cord injury, Lasers Surg. Med. 36 (3) (2005) 171-185.

[32] M. Niemz, Laser-tissue Interactions. Fundamentals and Applications, 3rd ed., Springer, 1996

[33] N. Yokoi, Y. Aizu, Depth measurement of a blood flow region based on speckle decorrelation, Opt. Rev. 22 (2) (2015) 365-373. 